\title{
Cardiac magnetic resonance derived left atrial strain after ST- elevation myocardial infarction: an independent prognostic indicator
}

\author{
Dhruv Nayyar ${ }^{1,2}$, Tuan Nguyen ${ }^{1,3}$, Faraz Pathan ${ }^{4,5}$, Giau Vo ${ }^{1}$, David Richards ${ }^{6}$, Liza Thomas ${ }^{3,6,7}$, \\ Hany Dimitri ${ }^{1,3}$, James Otton ${ }^{1,3}$
}

${ }^{1}$ Cardiology Department, Liverpool Hospital, Sydney, NSW, Australia; ${ }^{2}$ School of Medicine, Western Sydney University, Sydney, NSW, Australia; ${ }^{3}$ South West Clinical School, University of New South Wales, Sydney, NSW, Australia; ${ }^{4}$ Cardiology Department, Nepean Hospital, Sydney, NSW, Australia; ${ }^{5}$ Charles Perkins Centre Sydney, Nepean Clinical School, University of Sydney, Sydney, NSW, Australia; ${ }^{6}$ Westmead Clinical School, University of Sydney, Sydney, NSW, Australia; ${ }^{7}$ Cardiology Department, Westmead Hospital, Sydney, NSW, Australia

Contributions: (I) Conception and design: J Otton, D Richards, L Thomas, H Dimitri; (II) Administrative support: None; (III) Provision of study materials or patients: T Nguyen, D Richards, L Thomas, H Dimitri; (IV) Collection and assembly of data: D Nayyar, T Nguyen; (V) Data analysis and interpretation: D Nayyar, T Nguyen, F Pathan, D Richards, L Thomas, H Dimitri, J Otton; (VI) Manuscript writing: All authors; (VII) Final approval of manuscript: All authors.

Correspondence to: Dr. James Otton. Cardiology Department, 75 Elizabeth St Liverpool, NSW 2170, Australia. Email: jotton@gmail.com.

Background: The prognostic value of cardiac magnetic resonance (CMR) derived left atrial (LA) strain, ejection fraction (LAEF) and indexed volumes $\left(\mathrm{LAVI}_{\max }\right.$ and $\mathrm{LAVI}_{\min }$ ) after ST-elevation myocardial infarction (STEMI) remains controversial. The aim of this study was to assess the relationship between LA function and major adverse cardiovascular events (MACE) after STEMI.

Methods: A total of 202 prospectively recruited patients who underwent CMR at median day 4 after STEMI had complete CMR data for feature tracking assessment. LA reservoir and booster strain were quantified based on the average of three independently repeated measurements.

Results: MACE occurred in 35 patients during a median follow up of 607 days. Patients with MACE had lower median LA reservoir strain (18.9\% vs. 29.4\%, $\mathrm{P}<0.001)$, LA booster strain $(9.4 \%$ vs. $13.0 \%, \mathrm{P}=0.002)$ and LAEF (41.5\% vs. 49.2\%, $\mathrm{P}<0.001$ ) than patients without MACE. Kaplan-Meier analyses demonstrated a difference in MACE between high- and low-risk groups for LA reservoir strain (cutoff 19.2\%, P<0.001), LA booster strain (cutoff 9.7\%, $\mathrm{P}<0.001$ ) and LAEF (cutoff $38.5 \%, \mathrm{P}<0.001$ ). The AUC increased from 0.713 (95\% CI: 0.608-0.818) for LVEF to 0.775 (95\% CI: 0.680-0.870) when LA reservoir strain was added to LVEF ( $\mathrm{P}=0.047)$. Univariate Cox regression analysis showed that all LA parameters had a significant effect on MACE, while multivariate analysis found LA reservoir strain was an independent predictor of MACE (HR 0.905; 95\% CI: 0.843-0.972, P=0.006).

Conclusions: CMR derived LA reservoir strain independently predicted MACE after STEMI when adjusted for standard risk measures.

Keywords: Left atrial function; left atrial strain; ST-elevation myocardial infarction (STEMI); prognosis; cardiac magnetic resonance (CMR)

Submitted Oct 26, 2020. Accepted for publication Jan 25, 2021.

doi: $10.21037 / \mathrm{cdt}-20-879$

View this article at: http://dx.doi.org/10.21037/cdt-20-879 


\section{Introduction}

Left atrial (LA) dilatation is an established marker of adverse outcomes in a range of cardiovascular conditions including ischemic heart disease and heart failure (1). Cardiac magnetic resonance (CMR) is considered the gold standard for assessing LA volumetric indices given its reproducibility and high spatial resolution (2). LA ejection fraction (LAEF) has emerged as a volumetric measure of global LA systolic function which correlates with left ventricular ejection fraction (LVEF) and infarct size in ST-elevation myocardial infarction (STEMI) (3). However, CMR derived LA volumes and LAEF have not shown additional prognostic value compared to existing markers of myocardial damage in predicting major adverse cardiovascular events (MACE) after STEMI $(3,4)$.

LA strain measures the extent of myocardial deformation and is less subject to loading conditions than volumetric indices (5). LA reservoir strain represents the phase of pulmonary venous return during ventricular systole. LA booster strain corresponds to active atrial contraction, and accounts for the late-diastolic augmentation in ventricular filling which is absent in atrial fibrillation (AF). CMR feature tracking allows fast and reproducible assessment of LA strain from routine cine images (6). LA reservoir strain has been shown as an independent prognostic marker after acute myocardial infarction (MI) (7), however further studies are needed to validate these findings. The aim of this study was to assess whether CMR derived LA strain and volumetric indices predicted MACE after STEMI. We hypothesized that impaired LA function would be associated with worse outcomes.

We present the study in accordance with the MDAR reporting checklist (available at http://dx.doi.org/10.21037/ cdt-20-879).

\section{Methods}

The study was conducted in accordance with the Declaration of Helsinki (as revised in 2013). The study was approved by the Human Research Ethics Committee at Concord Hospital, Sydney Australia (HREC/11/ CRGH/224; approval CH62/6/2011-151) and informed consent was taken from all the patients.

\section{Study population}

We performed a secondary analysis on a cohort of prospectively recruited consecutive STEMI patients from May 2012 to June 2014 at a large tertiary referral centre treated with primary percutaneous coronary intervention (PCI), rescue PCI, or successful thrombolysis followed by non-emergent PCI (8). Exclusion criteria were age $<18$ years or $>85$ years, severe chronic kidney disease (eGFR $<30 \mathrm{~mL} / \mathrm{min} / 1.73 \mathrm{~m}^{2}$ or renal replacement therapy), previous $\mathrm{AF}$, known cardiomyopathy, prior cardiac surgery, severe claustrophobia, gadolinium allergy, and ferrous metallic implants. Patient follow-up was conducted for up to two years post-STEMI.

\section{CMR protocol}

A detailed protocol with the specific CMR parameters used has previously been described (8). Briefly, patients underwent CMR within 7 days post-STEMI using a commercially available $1.5 \mathrm{~T}$ MRI scanner (Siemens Symphony, Germany). A standard multisequence protocol was used during breath-hold. A 6-channel body array and spine coil were used. Retrospective vector ECG gating was used for cardiac synchronization. Cine images, using a steady state free precession pulse sequence, were acquired in standard short- and long-axis views. Late gadolinium enhancement images were obtained 8 to 10 minutes after a bolus injection of $0.1 \mathrm{mmol} / \mathrm{kg}$ gadoteric acid (Dotarem, Guerbet, France).

\section{CMR analysis}

All CMR analysis was performed offline using commercially available software (CVI42 version 5.11, Circle Cardiovascular Imaging, Calgary, Canada). LVEF, infarct scar size, microvascular obstruction (MVO) and myocardial salvage index (MSI) were measured as previously reported (8). Infarct size was defined as the hyper-enhanced area with signal intensity threshold $\geq 5$ standard deviation (SD) above a region of interest of normal 'nulled' myocardium and expressed as a percentage of the total left ventricular (LV) mass (9-11). MVO was determined by manual contouring of areas of hypo-enhancement with surrounding hyperenhanced myocardium on the delayed enhancement inversion recovery sequences $(9,12)$. Area at risk was defined as the volume of hyper-enhanced myocardium $(\geq 2 \mathrm{SD}$ of the region of interest) on T2 short tau inversion recovery sequences, divided by the total myocardial volume. MSI was calculated as: (area at risk - infarct scar size)/area at risk (9).

LA volumetric indices were measured by manually 

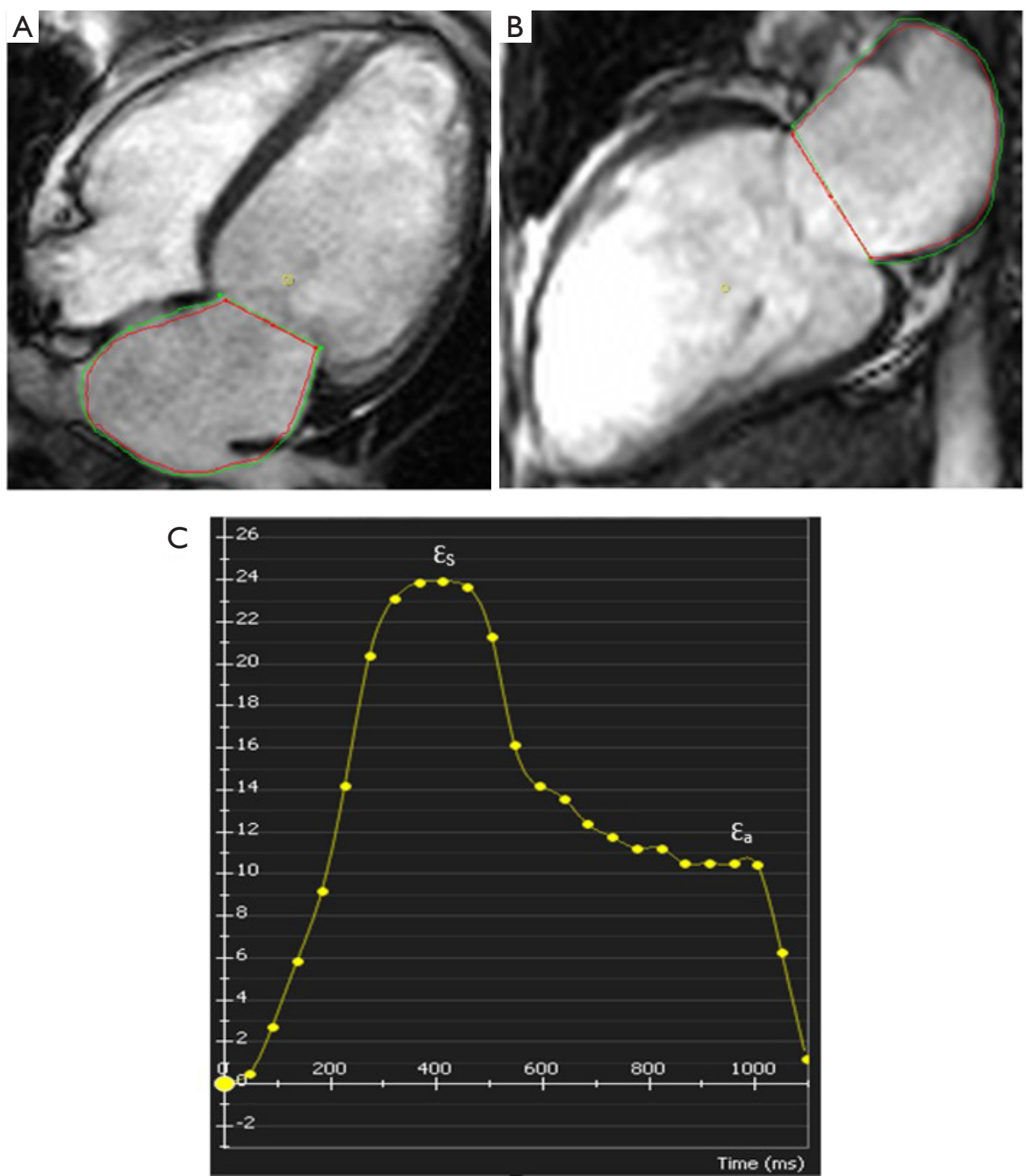

Figure 1 LA strain by CMR feature tracking. (A) Long axis four-chamber view, (B) long axis two-chamber view and (C) LA strain curve. The red line is the endocardial curve and the green line is the epicardial curve. $\varepsilon_{S}$, LA reservoir strain; $\varepsilon_{\mathrm{a}}$, LA booster strain. LA, Left atrial; $\mathrm{CMR}$, cardiac magnetic resonance.

tracing the LA endocardial border in end-systole and end-diastole in long-axis two- and four-chamber views. Maximum and minimum LA volumes were calculated based on the biplane area-length method (13) and indexed to body surface area $\left(\mathrm{LAVI}_{\max }\right.$ and $\left.\mathrm{LAVI}_{\min }\right)$. LAEF was defined as: $\left[\left(\mathrm{LAVI}_{\max }-\mathrm{LAVI}_{\min }\right) / \mathrm{LAVI}_{\max }\right] \times 100$.

LA strain was performed using CVI42 Tissue Tracking software (Circle Cardiovascular Imaging v5.11, Calgary, Canada). LA endocardial and epicardial borders were manually traced in end-systole in long-axis two- and four- chamber views. An automated tracking algorithm was applied, and manual adjustments were performed as needed to attain optimal wall tracking. The strain values for each tissue point were automatically derived by the software and were represented as a strain curve from which LA reservoir strain and LA booster strain were recorded (Figure 1). LA strain values were calculated based on the average of three independently repeated measurements.

LA volumetric and strain analysis was performed by one experienced observer blinded to clinical and CMR data. 


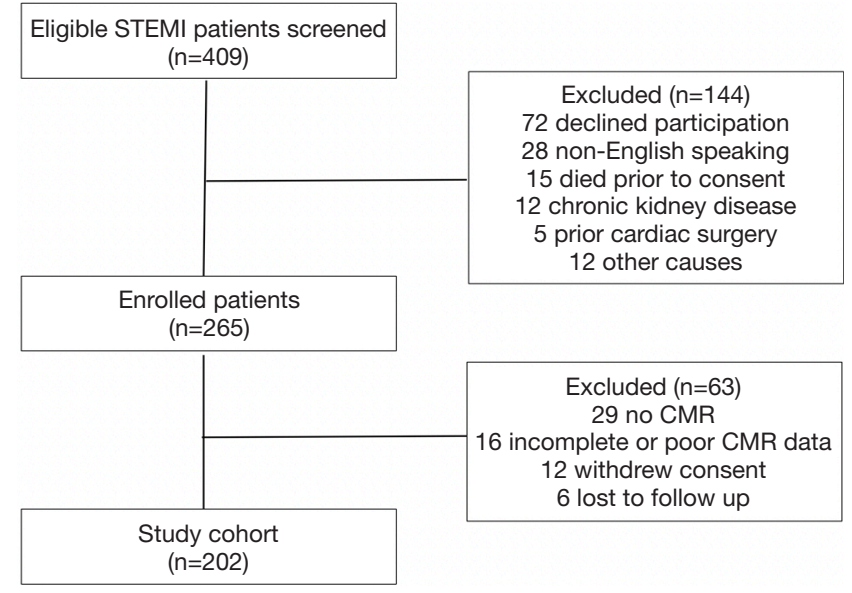

Figure 2 Study flowchart. CMR, cardiac magnetic resonance; STEMI, ST-elevation myocardial infarction.

Inter-observer and intra-observer variabilities were assessed in 20 random healthy subjects by two investigators, blinded to the first set of measurements.

\section{Diastolic function assessment}

Diastolic parameters were evaluated by contemporaneously performed transthoracic echocardiography using established criteria $(14,15)$ to categorise patients to a diastolic function grade $(0=$ normal, $1=$ impaired relaxation, $2=$ pseudonormal, $3=$ restrictive filling pattern) (16).

\section{Clinical endpoints}

The primary endpoint of MACE was a composite of allcause mortality, reinfarction, new or worsening heart failure, stroke, and sustained ventricular arrhythmias. For patients with more than one MACE, the primary endpoint was determined as time to first event. Detailed outcome definitions have been reported previously (16).

\section{Statistical analysis}

Continuous data with normal distribution are presented as mean \pm SD. Non-normally distributed variables are reported as median and interquartile range (IQR). Categorical variables are presented as frequencies and percentages. Comparison between groups was performed using the Student's $t$-test (normally distributed) and the Mann Whitney U-test (non-normally distributed) continuous variables, and the Chi-squared test for categorical variables. Correlation of normally distributed parameters was evaluated by Pearson's correlation coefficient and nonnormally distributed parameters with Spearman's rank correlation coefficient.

Receiver operator characteristic analyses were performed and area under the curve (AUC) calculated and compared using the De Long test. For evaluating the primary endpoint, Kaplan-Meier survival analysis was performed, and differences were assessed by the log-rank test. Cutoffs for high-risk groups were determined by the Youden index (17). Univariate and multivariate Cox proportional hazards regression models were performed to calculate hazard ratios (HR) with corresponding $95 \%$ confidence intervals (CI). The multivariate model comprised variables with $\mathrm{P}<0.05$ at univariate analysis. Inter-observer and intraobserver variabilities were assessed by intraclass correlation coefficients (ICC) and coefficients of variation (COV).

A $P$ value of $<0.05$ was considered statistically significant. Statistical analyses were performed using SPSS version 24.0 (IBM SPSS, IBM Corporation, Armonk, NY, USA) and MedCalc version 19.2.0 (MedCalc Software Ltd., Ostend, Belgium).

\section{Results}

Four hundred and nine consecutive STEMI patients were screened during the study period, of which 265 were enrolled; 29 patients could not undergo CMR, 16 had incomplete CMR data or poor image quality for feature tracking assessment, 12 withdrew consent, and 6 were lost to follow-up, resulting in 202 patients being included in this study (Figure 2).

The clinical characteristics of the study group are shown in Table 1. There were 174 (86.1\%) males in the cohort with mean age $56 \pm 11$ years. CMR was performed at median day 4 (IQR day 2-7). Over a median follow up 607 days (IQR 438-730 days), there were 42 primary endpoint events (death $\mathrm{n}=3$, reinfarction $\mathrm{n}=16$, readmission due to congestive heart failure $n=10$, ventricular arrhythmia $n=9$ and stroke $\mathrm{n}=4)$ in $35(17.3 \%)$ patients.

Patients with MACE were older (62 vs. 56 years, $\mathrm{P}<0.001)$ and more frequently had diabetes $(40 \%$ vs. $16 \%$, $\mathrm{P}=0.001)$, prior $\mathrm{MI}(20 \%$ vs. $6 \%, \mathrm{P}=0.007)$, three-vessel disease $(31 \%$ vs. $3 \%, \mathrm{P}<0.001)$ and anterior $\mathrm{MI}(71 \%$ vs. $49 \%, \mathrm{P}=0.019)$ compared to patients without MACE. TIMI risk score and peak high sensitivity troponin $\mathrm{T}$ (hs $\mathrm{Tn} \mathrm{T}$ ) were higher in patients with MACE than patients without 
Table 1 Baseline characteristics

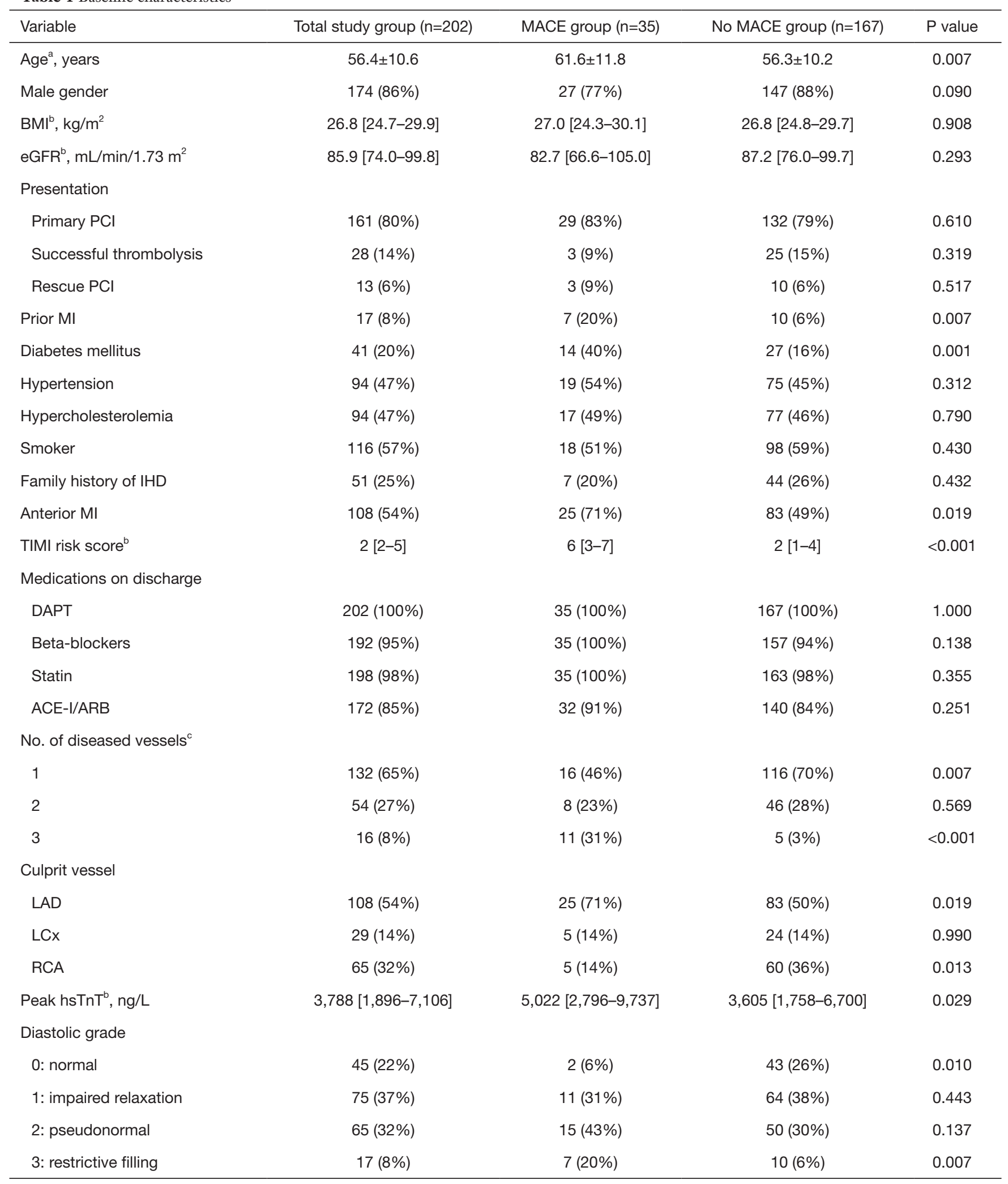

Table 1 (continued) 
Table 1 (continued)

\begin{tabular}{|c|c|c|c|c|}
\hline Variable & Total study group $(n=202)$ & MACE group $(n=35)$ & No MACE group $(n=167)$ & $P$ value \\
\hline \multicolumn{5}{|l|}{ Mitral regurgitation } \\
\hline None & 165 (82\%) & $24(69 \%)$ & $141(84 \%)$ & 0.027 \\
\hline Mild & $32(16 \%)$ & $9(26 \%)$ & $23(14 \%)$ & 0.079 \\
\hline Moderate & $4(2 \%)$ & $1(3 \%)$ & $3(2 \%)$ & 0.682 \\
\hline \multicolumn{5}{|l|}{ CMR characteristics } \\
\hline LVEF$^{a}, \%$ & $46.1 \pm 9.9$ & $39.0 \pm 11.2$ & $47.6 \pm 8.9$ & $<0.001$ \\
\hline Presence of MVO & $91(45 \%)$ & $22(63 \%)$ & $69(41 \%)$ & 0.020 \\
\hline MVO size ${ }^{\mathrm{b}}, \%$ & $0.00[0.00-0.48]$ & $0.12[0.00-1.04]$ & $0.00[0.00-0.42]$ & 0.026 \\
\hline
\end{tabular}

${ }^{a}$, mean $\pm \mathrm{SD} ;{ }^{b}$, median [interquartile range]; ${ }^{\circ}$, coronary artery stenosis $\geq 70 \%$. BMI, body mass index; eGFR, estimated glomerular filtration rate; $\mathrm{PCl}$, percutaneous coronary intervention; MI, myocardial infarction; IHD, ischemic heart disease; DAPT, dual antiplatelet therapy; ACE-I, angiotensin converting enzyme inhibitor; ARB, angiotensin receptor blocker; TIMI, thrombolysis in myocardial infarction; LAD, left anterior descending artery; LCx, left circumflex artery; RCA, right coronary artery; hsTnT, high sensitivity troponin T; LVEF, left ventricular ejection fraction; MVO, microvascular obstruction; MSI, myocardial salvage index; MACE, major adverse cardiovascular events.

MACE ( $\mathrm{P}<0.001$ and $\mathrm{P}=0.029$, respectively). Patients with MACE more frequently had grade 3 diastolic dysfunction than patients without MACE (20\% vs. 6\%, P=0.007). Patients with MACE had lower LVEF (39.0\% vs. $47.6 \%$, $\mathrm{P}<0.001)$ and MSI (68vs. 77, $\mathrm{P}<0.001$ ), and larger MVO size $(0.12 \%$ vs. $0.00 \%, \mathrm{P}=0.026)$ and infarct scar size $(13.7 \%$ vs. $8.4 \%, \mathrm{P}<0.001)$ than patients without MACE.

Patients with MACE had lower LA reservoir strain (18.9\% vs. 29.4\%, $\mathrm{P}<0.001)$, LA booster strain $(9.4 \%$ vs. $13.0 \%, \mathrm{P}=0.002)$ and LAEF $(41.5 \%$ vs. $49.2 \%, \mathrm{P}<0.001)$, and higher $\mathrm{LAVI}_{\max }\left(43.5\right.$ vs. $\left.38.6 \mathrm{~mL} / \mathrm{m}^{2}, \mathrm{P}=0.019\right)$ and LAVI $_{\text {min }}\left(23.7\right.$ vs. $\left.19.3 \mathrm{~mL} / \mathrm{m}^{2}, \mathrm{P}<0.001\right)$ compared to patients without MACE. Patients with LVEF $\leq 40 \%$ had significantly lower LA reservoir strain $(21.4 \%$ vs. $30.0 \%$, $\mathrm{P}<0.001)$, LA booster strain $(11.3 \%$ vs. $13.1 \%, \mathrm{P}=0.021)$ and LAEF $(43.3 \%$ vs. $50.3 \%, \mathrm{P}<0.001)$, and higher $\mathrm{LAVI}_{\min }$ (21.4 vs. $19.5 \mathrm{~mL} / \mathrm{m}^{2}, \mathrm{P}=0.003$ ) than patients with $\mathrm{LVEF}$ $>40 \%$. Patients with MVO had significantly lower LA reservoir strain $(26.1 \%$ vs. $31.0 \%, \mathrm{P}<0.001)$, LA booster strain $(11.7 \%$ vs. $13.0 \%, \mathrm{P}=0.021)$ and LAEF $(44.8 \%$ vs. $51.2 \%, \mathrm{P}<0.001)$, and higher $\mathrm{LAVI}_{\min }\left(21.3\right.$ vs. $18.9 \mathrm{~mL} / \mathrm{m}^{2}$, $\mathrm{P}=0.012)$ than patients without MVO. Patients with diastolic dysfunction had significantly lower LA reservoir strain $(27.4 \%$ vs. $30.3 \%, \mathrm{P}=0.028)$ than patients with normal diastolic function.
LA reservoir strain and LAEF had a weak to moderate positive correlation with LVEF and MSI, and inverse correlation with infarct scar size and peak hsTnT (Table 2).

AUC analyses showed LA reservoir strain (AUC 0.769; 95\% CI: 0.676-0.861), LA booster strain (AUC 0.684; 95\% CI: 0.558-0.810), LAEF (AUC 0.698; 95\% CI:

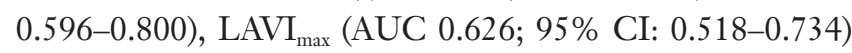
and $\mathrm{LAVI}_{\min }$ (AUC 0.695; 95\% CI: 0.591-0.799) provided accurate prediction of MACE. The addition of LA reservoir strain to LVEF resulted in a significant increase in AUC from 0.713 (95\% CI: 0.608-0.818) for LVEF alone to 0.775 (95\% CI: 0.680-0.870) for LVEF and LA reservoir strain $(\mathrm{P}=0.047)$ (Figure 3).

Kaplan-Meier analysis showed a difference in MACE between high- and low-risk groups for LA reservoir strain (cutoff 19.2\%, $\mathrm{P}<0.001$ ), LA booster strain (cutoff 9.7\%, $\mathrm{P}<0.001$ ), LAEF (cutoff $38.5 \%, \mathrm{P}<0.001$ ), $\mathrm{LAVI}_{\max }$ (cutoff $47.5 \mathrm{~mL} / \mathrm{m}^{2}, \mathrm{P}=0.001$ ), and $\mathrm{LAVI}_{\min }$ (cutoff $22.6 \mathrm{~mL} / \mathrm{m}^{2}$, $\mathrm{P}<0.001$ ) (Figure 4).

Univariate Cox regression analysis showed all LA parameters had a significant effect on MACE (Table 3) including LA reservoir strain (HR 0.892; $95 \%$ CI: 0.852-0.935; $\mathrm{P}<0.001$ ), LA booster strain (HR 0.838; 95\% CI: 0.745-0.944; $\mathrm{P}=0.004$ ), LAEF (HR 0.938; 95\% CI: $0.909-0.967 ; \mathrm{P}<0.001), \mathrm{LAVI}_{\max }(\mathrm{HR} 1.041 ; 95 \%$ 
Table 2 Correlation coefficient analysis

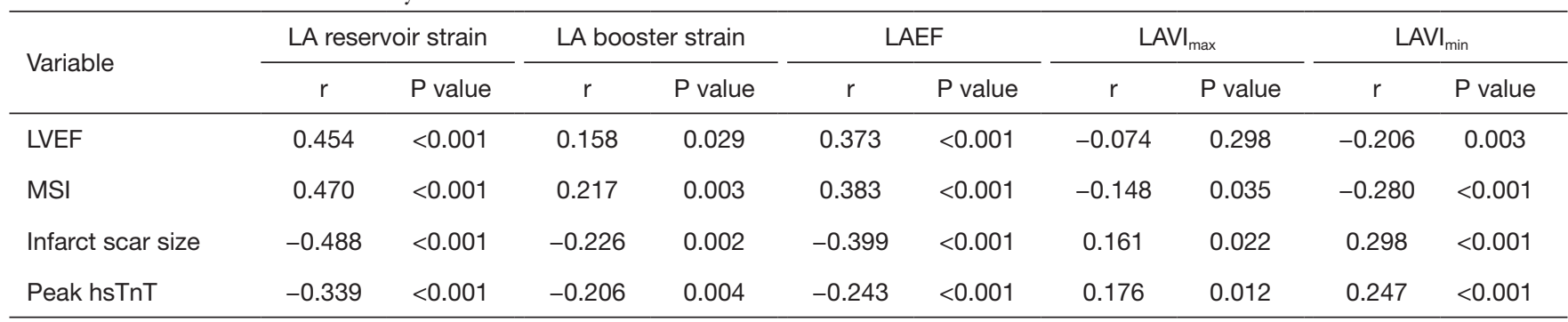

LVEF, left ventricular ejection fraction; MSI, myocardial salvage index; hsTnT, high sensitivity troponin T; LA, left atrial; LAEF, left atrial ejection fraction; $L A V I_{\max }$, maximum indexed left atrial volume; $L A V I_{\min }$, minimum indexed left atrial volume.

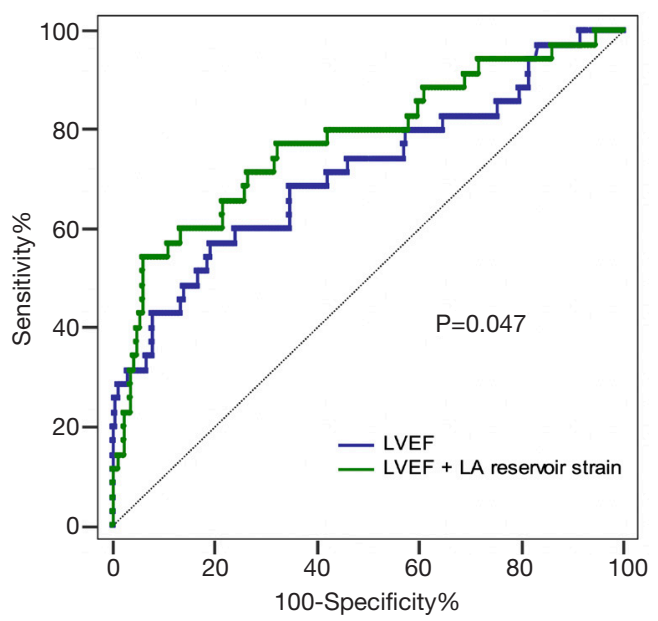

Figure 3 Receiver operator characteristic curve evaluating LVEF compared to LA reservoir strain in addition to LVEF in predicting major adverse cardiovascular events. LVEF, left ventricular ejection fraction; LA, left atrial.

CI: $1.012-1.072 ; \mathrm{P}=0.005)$ and $\mathrm{LAVI}_{\min }(\mathrm{HR} 1.059 ; 95 \%$ CI: $1.027-1.093 ; \mathrm{P}<0.001)$. Multivariate Cox regression modelling demonstrated that LA reservoir strain (HR 0.920; 95\% CI: 0.873-0.969; $\mathrm{P}=0.002$ ), LA booster strain (HR 0.872; 95\% CI: 0.774-0.983; $\mathrm{P}=0.025$ ), LAEF (HR 0.959; 95\% CI: 0.928-0.992; $\mathrm{P}=0.015)$ and $\mathrm{LAVI}_{\min }(\mathrm{HR} 1.040$; 95\% CI: $1.007-1.073 ; \mathrm{P}=0.016)$ were predictors of MACE independent of LVEF (Table 4). LA reservoir strain was also adjusted for all variables with $\mathrm{P}<0.05$ at univariate analysis including age, previous MI, diabetes mellitus, anterior MI, three-vessel disease, TIMI risk score, peak hsTnT, LVEF, infarct scar size and diastolic dysfunction. MSI was excluded because it demonstrated collinearity with infarct scar size. This multivariate model showed that LA reservoir strain (HR 0.905; 95\% CI: 0.843-0.972; $\mathrm{P}=0.006$ ), three-vessel disease (HR 22.987; 95\% CI: 4.256-124.158; $\mathrm{P}<0.001$ ) and
TIMI risk score (HR 1.497; 95\% CI: 1.113-2.013; $\mathrm{P}=0.008$ ) were independent predictors of MACE.

One way ANOVA showed no significant difference in mean LA reservoir strain between normal diastolic function $(29.4 \% \pm 7.8 \%)$, grade 1 diastolic dysfunction $(27.1 \% \pm 8.3 \%)$ and grade 2 diastolic dysfunction $(27.9 \% \pm 9.4 \%)$, however were significantly higher than patients with grade 3 diastolic dysfunction $(15.8 \% \pm 9.1 \%, \mathrm{P}<0.001$ for all).

Intra-observer reproducibility was excellent for LA reservoir strain (ICC 0.976 and 0.989 , COV $7.1 \%$ and $6.9 \%$ ) and LA booster strain (ICC 0.951 and 0.948 , COV $6.2 \%$ and $8.8 \%)$ for both investigators. Inter-observer reproducibility was excellent for LA reservoir strain (ICC 0.948, COV 11.7\%) and LA booster strain (ICC 0.977, COV 8.1\%).

\section{Discussion}

Our study shows that impaired LA reservoir strain and LA booster strain are associated with worse outcomes after STEMI. There was a significant increase in MACE for high-risk groups with LA reservoir strain below 19.2\% and LA booster strain below 9.7\%. This may be because preserved LA function initially compensates for the increased LV chamber stiffness and filling pressures postMI (18), and loss of this compensatory mechanism from atrial non-compliance results in impaired LV filling $(19,20)$. We found that LA reservoir strain and LA booster strain predicted MACE independent of LVEF. Whilst identifying the possibility of model overfitting, we found that LA reservoir strain was also an independent predictor of MACE after adjusting for established clinical and imaging risk measures which were significant at univariate analysis.

Furthermore, AUC analysis demonstrated a significant improvement in MACE prediction when LA reservoir strain was added to LVEF. This is an important finding considering 

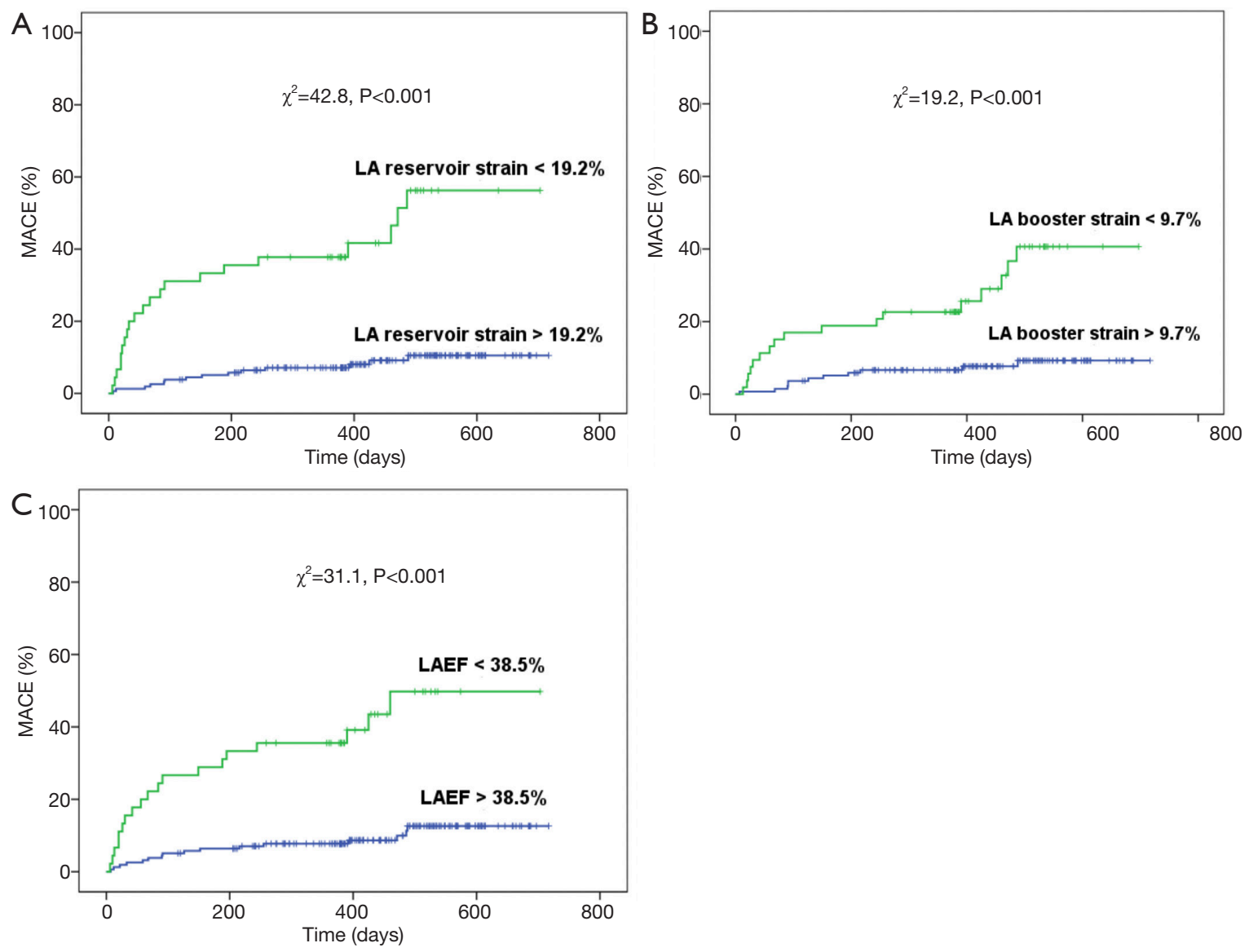

Figure 4 Kaplan Meier survival curves for (A) LA reservoir strain, (B) LA booster strain and (C) LAEF with major adverse cardiovascular events after dichotomisation, as calculated by the Youden index. LA, left atrial; LAEF, left atrial ejection fraction.

LVEF is currently the most widely used risk predictor post STEMI in guiding heart failure therapy. By comparison, Ledwoch et al. found that LAEF did not have an additive value in terms of MACE prediction after acute MI over and above LVEF (3), highlighting the utility of phasic measures such as LA reservoir strain over volumetric indices.

Our study provides external validation to the findings of Schuster et al. (7) in a patient cohort with a higher MACE rate and longer follow up duration. One limitation of the study by Schuster et al. was that assessment of diastolic function was not performed, and hence the authors could not assess the effect of diastolic dysfunction on LA strain or exclude the possibility that changes in atrial function reflected ventricular diastology (7). By comparison, our study is novel because we have assessed diastology with a paired CMR and transthoracic echocardiogram for each patient and have adjusted for diastolic dysfunction and mitral regurgitation severity. This is particularly important because recent studies have demonstrated a linear decrease in LA reservoir strain with increasing grades of diastolic dysfunction $(21,22)$, and significant diastolic dysfunction has been shown to be an independent predictor of adverse outcomes post MI (23). We found similar cutoff values for LA reservoir strain (19.2\% vs. 18.8\%) and LA booster strain (9.7\% vs. $10.1 \%)$ to Schuster et al. despite using different vendors for feature tracking analysis (CVI42 vs. TomTec Imaging Systems) (7), demonstrating the robustness of the derived strain values. In contrast to LA strain, which is relatively independent of atrial size, $\mathrm{LAVI}_{\max }$ was not an independent predictor of MACE when adjusted for LVEF. LA reservoir strain also demonstrated a stronger correlation with established markers of myocardial damage including LVEF, infarct scar size, MSI and peak hsTnT compared to $\mathrm{LAVI}_{\min }$ and $\mathrm{LAVI}_{\max }$. Similar to Ledwoch et al., we found that there was a modest positive correlation between LAEF and LVEF ( $\mathrm{r}=0.373)$, and a modest inverse correlation 
Table 3 Univariate Cox regression analysis of predictors for major adverse cardiovascular events

\begin{tabular}{|c|c|c|}
\hline Variable & Univariable hazard ratio & $P$ value \\
\hline Age & $1.051(1.013-1.091)$ & 0.008 \\
\hline Male gender & $0.459(0.184-1.149)$ & 0.096 \\
\hline Diabetes & 3.457 (1.566-7.631) & 0.002 \\
\hline Hypertension & $1.457(0.701-3.028)$ & 0.314 \\
\hline Smoking & $0.745(0.359-1.548)$ & 0.431 \\
\hline Anterior MI & $2.530(1.144-5.595)$ & 0.022 \\
\hline Previous MI & 3.925 (1.379-11.174) & 0.010 \\
\hline Three-vessel disease & $14.850(4.746-46.467)$ & $<0.001$ \\
\hline TIMI risk score & $1.717(1.413-2.085)$ & $<0.001$ \\
\hline Peak hsTnT & $1.125(1.038-1.219)$ & 0.004 \\
\hline Diastolic dysfunction & $5.722(1.317-24.856)$ & 0.02 \\
\hline Mitral regurgitation & $3.313(0.533-20.609)$ & 0.199 \\
\hline LVEF & $0.908(0.869-0.948)$ & $<0.001$ \\
\hline MVO size & $1.208(0.996-1.464)$ & 0.054 \\
\hline Infarct scar size & $1.101(1.049-1.156)$ & $<0.001$ \\
\hline MSI & $0.958(0.935-0.981)$ & $<0.001$ \\
\hline LA reservoir strain & $0.892(0.852-0.935)$ & $<0.001$ \\
\hline LA booster strain & $0.838(0.745-0.944)$ & 0.004 \\
\hline LAEF & $0.938(0.909-0.967)$ & $<0.001$ \\
\hline$L A V I_{\max }$ & $1.041(1.012-1.072)$ & 0.005 \\
\hline $\mathrm{LAVI}_{\min }$ & $1.059(1.027-1.093)$ & $<0.001$ \\
\hline
\end{tabular}

Data in parentheses are 95\% confidence intervals. Diastolic dysfunction included impaired relaxation, pseudonormal and restrictive filling patterns. Mitral regurgitation included moderate and severe mitral regurgitation. MI, myocardial infarction; TIMI, thrombolysis in myocardial infarction; hsTnT, high sensitivity troponin T; LVEF, left ventricular ejection fraction; MVO, microvascular obstruction; MSI, myocardial salvage index; LA, left atrial; LAEF, left atrial ejection fraction; $L A V I_{\max }$, maximum indexed left atrial volume; $L A \bigvee I_{\text {min }}$, minimum indexed left atrial volume.

between LAEF and infarct scar size ( $\mathrm{r}=-0.399)$ (3). This may be because volumetric indices are subject to geometrical assumptions and are load dependent, and LA reservoir strain better reflects intrinsic LA function (20,24). LA reservoir strain reflects atrial compliance and to a lesser extent, atrial contractility and relaxation, modulated by descent of the $\mathrm{LV}$ base during systole $(1,18)$. Volumetric indices may also be limited by lower sensitivity in early disease states compared with LA strain (25). $\mathrm{LAVI}_{\max }$ is predominantly a marker of
Table 4 Multivariate Cox regression modelling of left atrial variables adjusted for left ventricular ejection fraction

\begin{tabular}{lcc}
\hline Variable & Multivariable hazard ratio & P value \\
\hline LA reservoir strain & $0.920(0.873-0.969)$ & 0.002 \\
LA booster strain & $0.872(0.774-0.983)$ & 0.025 \\
LAEF & $0.959(0.928-0.992)$ & 0.015 \\
LAVI $_{\max }$ & $1.030(0.999-1.061)$ & 0.055 \\
LAVI $_{\min }$ & $1.040(1.007-1.073)$ & 0.016 \\
\hline
\end{tabular}

Data in parentheses are 95\% confidence intervals. Each hazard ratio is derived from a separate model containing the LA parameter and left ventricular ejection fraction. LA, left atrial; $L A E F$, left atrial ejection fraction; $L A V I_{\max }$, maximum indexed left atrial volume; $\mathrm{LAVI}_{\min }$, minimum indexed left atrial volume.

chronically elevated LV filling pressure, whereas $\mathrm{LAVI}_{\min }$ is more sensitive to changes in atrial afterload and atrial elastance (1). Lønborg et al. showed that $\mathrm{LAVI}_{\max }$ is not associated with acute LV function but determined by preexisting conditions, whilst $\mathrm{LAVI}_{\min }$ is determined by acute changes in LV function such as acute stunning and infarct size as well as pre-existing conditions (4).

We postulate that LA reservoir strain may potentially have a role in improving risk stratification post-STEMI in the future, although further validation studies are needed. It is promising that the technique does not require additional CMR scanning time and offline analysis can be performed efficiently with high inter-observer and intra-observer reproducibility.

The limitations of our study include the relatively small number of primary endpoint events which makes it difficult to develop a comprehensive risk prediction model. Second, we included patients who were able to tolerate CMR and this may have excluded some of the most critically ill patients, which has implications on MACE rates. Third, the cutoff values for high-risk groups for each LA parameter were not compared to a control group. Fourth, the assessment of diastolic function was performed based on previous criteria, which have subsequently been updated. Finally, the exclusion of patients with AF may have limited the prognostic role of LA booster strain which is absent in AF.

In conclusion, impaired LA function predicted MACE independent of LVEF and may have a future role in improving risk stratification after STEMI.

\section{Acknowledgments}

Funding: None. 


\section{Footnote}

Reporting Checklist: The authors have completed the Materials Design Analysis Reporting (MDAR) checklist. Available at http://dx.doi.org/10.21037/cdt-20-879

Data Sharing Statement: Available at http://dx.doi. org/10.21037/cdt-20-879

Peer Review File: Available at http://dx.doi.org/10.21037/ cdt-20-879

Conflicts of Interest: All authors have completed the ICMJE uniform disclosure form (available at http://dx.doi. org/10.21037/cdt-20-879). The authors have no conflicts of interest to declare.

Ethical Statement: The authors are accountable for all aspects of the work in ensuring that questions related to the accuracy or integrity of any part of the work are appropriately investigated and resolved. The study was conducted in accordance with the Declaration of Helsinki (as revised in 2013). The study was approved by the Human Research Ethics Committee at Concord Hospital, Sydney Australia (HREC/11/CRGH/224; approval CH62/6/2011$151)$ and informed consent was taken from all the patients.

Open Access Statement: This is an Open Access article distributed in accordance with the Creative Commons Attribution-NonCommercial-NoDerivs 4.0 International License (CC BY-NC-ND 4.0), which permits the noncommercial replication and distribution of the article with the strict proviso that no changes or edits are made and the original work is properly cited (including links to both the formal publication through the relevant DOI and the license). See: https://creativecommons.org/licenses/by-nc-nd/4.0/.

\section{References}

1. Hoit BD. Left Atrial Size and Function: Role in Prognosis. J Am Coll Cardiol 2014;63:493-505.

2. Maceira AM, Cosín-Sales J, Roughton M, et al. Reference left atrial dimensions and volumes by steady state free precession cardiovascular magnetic resonance. J Cardiovasc Magn Reson 2010;12:65.

3. Ledwoch J, Stiermaier T, Fuernau G, et al. Prognostic Value and Determinants of CMR-Derived Left Atrial Function Assessed in STEMI. JACC Cardiovasc Imaging
2018;11:148-50.

4. Lønborg JT, Engstrøm T, Møller JE, et al. Left atrial volume and function in patients following ST elevation myocardial infarction and the association with clinical outcome: a cardiovascular magnetic resonance study. Eur Heart J Cardiovasc Imaging 2013;14:118-27.

5. Thomas L, Marwick TH, Popescu BA, et al. Left Atrial Structure and Function, and Left Ventricular Diastolic Dysfunction: JACC State-of-the-Art Review. J Am Coll Cardiol 2019;73:1961-77.

6. Schuster A, Hor KN, Kowallick JT, et al. Cardiovascular Magnetic Resonance Myocardial Feature Tracking. Circ Cardiovasc Imaging 2016;9:e004077.

7. Schuster A, Backhaus SJ, Stiermaier T, et al. Left Atrial Function with MRI Enables Prediction of Cardiovascular Events after Myocardial Infarction: Insights from the AIDA STEMI and TATORT NSTEMI Trials. Radiology 2019;293:292-302.

8. Nguyen TL, Phan JAK, Hee L, et al. High-sensitivity troponin $\mathrm{T}$ predicts infarct scar characteristics and adverse left ventricular function by cardiac magnetic resonance imaging early after reperfused acute myocardial infarction. Am Heart J 2015;170:715-25.e2.

9. Eitel I, de Waha S, Wöhrle J, et al. Comprehensive Prognosis Assessment by CMR Imaging After ST-Segment Elevation Myocardial Infarction. J Am Coll Cardiol 2014;64:1217-26.

10. Schulz-Menger J, Bluemke DA, Bremerich J, et al. Standardized image interpretation and post processing in cardiovascular magnetic resonance: Society for Cardiovascular Magnetic Resonance (SCMR) Board of Trustees Task Force on Standardized Post Processing. J Cardiovasc Magn Reson 2013;15:35.

11. Flett AS, Hasleton J, Cook C, et al. Evaluation of Techniques for the Quantification of Myocardial Scar of Differing Etiology Using Cardiac Magnetic Resonance. JACC Cardiovasc Imaging 2011;4:150-6.

12. Mayr A, Klug G, Schocke M, et al. Late microvascular obstruction after acute myocardial infarction: Relation with cardiac and inflammatory markers. Int J Cardiol 2012;157:391-6.

13. Lang RM, Bierig M, Devereux RB, et al. Recommendations for Chamber Quantification: A Report from the American Society of Echocardiography\&\#x2019;s Guidelines and Standards Committee and the Chamber Quantification Writing Group, Developed in Conjunction with the European Association of Echocardiography, a Branch of the European Society of Cardiology. J Am Soc 
Echocardiogr 2005;18:1440-63.

14. Møller JE, Pellikka PA, Hillis GS, et al. Prognostic Importance of Diastolic Function and Filling Pressure in Patients With Acute Myocardial Infarction. Circulation 2006;114:438-44.

15. Nagueh SF, Appleton CP, Gillebert TC, et al. Recommendations for the Evaluation of Left Ventricular Diastolic Function by Echocardiography. J Am Soc Echocardiogr 2009;22:107-33.

16. Nguyen TL, French JK, Hogan J, et al. Prognostic value of high sensitivity troponin $\mathrm{T}$ after ST-segment elevation myocardial infarction in the era of cardiac magnetic resonance imaging. Eur Heart J Qual Care Clin Outcomes 2016;2:164-71.

17. Fluss R, Faraggi D, Reiser B. Estimation of the Youden Index and its Associated Cutoff Point. Biom J 2005;47:458-72.

18. Barbier P, Solomon SB, Schiller NB, et al. Left Atrial Relaxation and Left Ventricular Systolic Function Determine Left Atrial Reservoir Function. Circulation 1999;100:427-36.

19. Wakami K, Ohte N, Asada K, et al. Correlation between Left Ventricular End-diastolic Pressure and Peak Left Atrial Wall Strain during Left Ventricular Systole. J Am

Cite this article as: Nayyar D, Nguyen T, Pathan F, Vo G, Richards D, Thomas L, Dimitri H, Otton J. Cardiac magnetic resonance derived left atrial strain after ST-elevation myocardial infarction: an independent prognostic indicator. Cardiovasc Diagn Ther 2021;11(2):383-393. doi: 10.21037/cdt-20-879
Soc Echocardiogr 2009;22:847-51.

20. Antoni ML, ten Brinke EA, Atary JZ, et al. Left atrial strain is related to adverse events in patients after acute myocardial infarction treated with primary percutaneous coronary intervention. Heart 2011;97:1332-7.

21. Singh A, Addetia K, Maffessanti F, et al. LA Strain for Categorization of LV Diastolic Dysfunction. JACC Cardiovasc Imaging 2017;10:735-43.

22. Frydas A, Morris DA, Belyavskiy E, et al. Left atrial strain as sensitive marker of left ventricular diastolic dysfunction in heart failure. ESC Heart Fail 2020;7:1956-65.

23. Prasad SB, Lin AK, Guppy-Coles KB, et al. Diastolic Dysfunction Assessed Using Contemporary Guidelines and Prognosis Following Myocardial Infarction. J Am Soc Echocardiogr 2018;31:1127-36.

24. Anwar AM, Soliman OII, Geleijnse ML, et al. Assessment of left atrial volume and function by realtime three-dimensional echocardiography. Int J Cardiol 2008;123:155-61.

25. Morris DA, Takeuchi M, Krisper M, et al. Normal values and clinical relevance of left atrial myocardial function analysed by speckle-tracking echocardiography: multicentre study. Eur Heart J Cardiovasc Imaging 2015;16:364-72. 